\title{
Graph invariants in the edge model
}

\author{
Alexander Schrijver ${ }^{1}$
}

Abstract. We sharpen the characterization of Szegedy of graph invariants

$$
f_{b}(G)=\sum_{\phi: E G \rightarrow[n]} \prod_{v \in V G} b(\phi(\delta(v))),
$$

where $b$ is a real-valued function defined on the collection of all multisubsets of $[n]:=\{1, \ldots, n\}$.

\section{Introduction}

Laci Lovász is a main inspirator of the new area of graph limits and graph connection matrices and their relations to graph parameters, partition functions, mathematical physics, reflection positivity, and extremal combinatorics. Prompted by Lovász's questions, Balázs Szegedy [5] characterized graph invariants in the 'edge model'. His proof is based on a highly original combination of methods from invariant theory and real algebraic geometry. It answers a question formulated in [1], which paper considers the corresponding 'vertex model'.

In this paper we give a sharpening of Szegedy's theorem and give a slightly shorter proof, although parts of our proof follow the scheme of Szegedy's proof. New elements of the present paper are the connections between linking of graphs and differentiation of polynomials and the use of a deep theorem of Procesi and Schwarz [4] in real invariant theory.

Let $\mathcal{G}$ be the collection of all finite graphs, where two graphs are considered to be the same if they are isomorphic. Graphs may have loops and multiple edges. Moreover, 'pointless' edges are allowed, that is, loops without a vertex. We use the notation

$$
[n]:=\{1, \ldots, n\}
$$

for any $n \in \mathbb{N}$, where $\mathbb{N}=\{0,1,2, \ldots\}$.

A graph invariant is any function $f: \mathcal{G} \rightarrow \mathbb{R}$. In this paper, as in Szegedy [5], we consider graph invariants obtained as follows. Let $n \in \mathbb{N}$ and let $A_{n}$ be the collection of all multisubsets of $[n]$, that is, of all multisets with elements from $[n]$. (So each element of $[n]$ has a 'multiplicity' in any $\alpha \in A_{n}$. There is a one-to-one relation between $A_{n}$ and $\mathbb{N}^{n}$, given by the multiplicities of $i \in[n]$ in a multiset $\alpha \in A_{n}$.)

For $b: A_{n} \rightarrow \mathbb{R}$ define $f_{b}: \mathcal{G} \rightarrow \mathbb{R}$ by

$$
f_{b}(G)=\sum_{\phi: E G \rightarrow[n]} \prod_{v \in V G} b(\phi(\delta(v)))
$$

\footnotetext{
${ }^{1}$ CWI and University of Amsterdam. Mailing address: CWI, Kruislaan 413, 1098 SJ Amsterdam, The Netherlands. Email: lex@cwi.nl.
} 
for $G \in \mathcal{G}$. Here $V G$ and $E G$ denote the sets of vertices and edges of $G$, respectively, $\delta(v)$ is the set of edges incident with $v$, and $\phi(\delta(v))$ is the multiset of $\phi$-values on $\delta(v)$, counting muytiplicities. (Actually, also $\delta(v)$ is a multiset, as loops at $v$ occur twice in $\delta(v)$.)

Several graph invariants are equal to $f_{b}$ for some appropriate $b$. For instance, the number of proper $n$-edge-colourings of a graph $G$ is equal to $f_{b}(G)$, where, for $\alpha \in A_{n}, b(\alpha)=1$ if all elements of $[n]$ have multiplicity 0 or 1 in $\alpha$, and $b(\alpha)=0$ otherwise. The number of perfect matchings in $G$ is equal to $f_{b}(G)$ for $n=2$ and $b(\alpha)=1$ if the multiplicity of 1 in $\alpha$ is equal to 1 , and $b(\alpha)=0$ otherwise. For more background, see de la Harpe and Jones [3] and Freedman, Lovász, and Schrijver [1].

We characterize which graph invariants $f$ satisfy $f=f_{b}$ for some $n \in \mathbb{N}$ and $b: A_{n} \rightarrow \mathbb{R}$, extending the characterizing of Szegedy [5]. We also prove that $f_{b}=f_{c}$ if and only if $c$ arises from $b$ by an orthogonal transformation. (Szegedy proved sufficiency here.)

\section{The characterization}

To describe the characterization, call a graph invariant $f$ multiplicative if $f\left(K_{0}\right)=1$ and $f(G H)=f(G) f(H)$ for any $G, H \in \mathcal{G}$. Here $K_{0}$ is the graph with no vertices and no edges, and $G H$ denotes the disjoint union of $G$ and $H$.

We also need the following operation. Let $u$ and $v$ be distinct vertices of a graph $G$, and let $\pi$ be a bijection from $\delta(u)$ to $\delta(v)$. (This obviously requires that $\operatorname{deg}(u)=\operatorname{deg}(v)$.) Let $G_{u, v, \pi}$ be the graph obtained as follows (where we consider the graph as topological space). Delete $u$ and $v$ from $G$, and for each $e \in \delta(u)$, reconnect $e$ to $\pi(e)$. So the open ends of $e$ and $\pi(e)$ are glued together with a new topological point (which however will not be a vertex). It might be that $e=\pi(e)$ (so $e$ connects $u$ and $v$ ), in which case we create a pointless loop.

We need a repeated application of this operation, denoted as follows. Let $u_{1}, v_{1}, \ldots, u_{k}, v_{k}$ be distinct vertices of graph $G$ and let $\pi_{i}: \delta\left(u_{i}\right) \rightarrow \delta\left(v_{i}\right)$ be a bijection, for each $i=1, \ldots, k$. Then we set

$$
G_{u_{1}, v_{1}, \pi_{1}, \ldots, u_{k}, v_{k}, \pi_{k}}:=\left(\cdots\left(G_{u_{1}, v_{1}, \pi_{1}}\right) \cdots\right)_{u_{k}, v_{k}, \pi_{k}}
$$

Now define the $\mathcal{G} \times \mathcal{G}$ matrix $M_{f, k}$ by

$$
\left(M_{f, k}\right)_{G, H}:=\sum_{u_{1}, v_{1}, \pi_{1}, \ldots, u_{k}, v_{k}, \pi_{k}} f\left((G H)_{u_{1}, v_{1}, \pi_{1}, \ldots, u_{k}, v_{k}, \pi_{k}}\right)
$$

for $G, H \in \mathcal{G}$, where the sum extends over all distinct $u_{1}, \ldots, u_{k} \in V G$, distinct $v_{1}, \ldots, v_{k} \in$ $V H$, and bijections $\pi_{i}: \delta_{G}\left(u_{i}\right) \rightarrow \delta_{H}\left(v_{i}\right)$, for $i=1, \ldots, k$.

Theorem 1. Let $f: \mathcal{G} \rightarrow \mathbb{R}$. Then $f=f_{b}$ for some $n \in \mathbb{N}$ and some $b: A_{n} \rightarrow \mathbb{R}$ if and only if $f$ is multiplicative and $M_{f, k}$ is positive semidefinite for each $k=0,1, \ldots$

The positive semidefiniteness of $M_{f, k}$ can be seen as a form of 'reflection positivity' of $f$. In Section 6, we derive Szegedy's characterization from Theorem 1. In Section 7 we prove that $b$ is uniquely determined by $f$, up to certain orthogonal transformations. 


\section{Some framework}

Let $\mathcal{Q}$ denote the collection of all formal real linear combinations $\sum_{G} \gamma_{G} G$ of graphs (with at most finitely many $\gamma_{G}$ nonzero). These are called quantum graphs. By taking the disjoint union $G H$ as multiplication, $\mathcal{Q}$ becomes a commutative algebra. The function $f$ can be extended linearly to $\mathcal{Q}$.

For $G, H \in \mathcal{G}$ and $k \in \mathbb{N}$, define the quantum graph $\lambda_{k}(G, H)$ by

$$
\lambda_{k}(G, H):=\sum_{u_{1}, v_{1}, \pi_{1}, \ldots, u_{k}, v_{k}, \pi_{k}}(G H)_{u_{1}, v_{1}, \pi_{1}, \ldots, u_{k}, v_{k}, \pi_{k}}
$$

where the sum is taken over the same set as in (4). We can extend $\lambda_{k}(G, H)$ linearly to a bilinear function $\mathcal{Q} \times \mathcal{Q} \rightarrow \mathcal{Q}$. As

$$
M_{f, k}(G, H)=f\left(\lambda_{k}(G, H)\right)
$$

the positive semidefiniteness of $M_{f, k}$ is equivalent to the fact that $f\left(\lambda_{k}(\gamma, \gamma)\right) \geq 0$ for each $\gamma \in \mathcal{Q}$.

For each $\alpha \in A_{n}$, we introduce a variable $x_{\alpha}$. For each $G \in \mathcal{G}$, define the following polynomial in $\mathbb{R}\left[x_{\alpha} \mid \alpha \in A_{n}\right]$ :

$$
p_{n}(G):=\sum_{\phi: E G \rightarrow[n]} \prod_{v \in V G} x_{\phi(\delta(v))} .
$$

So $f_{b}(G)=p_{n}(G)(b)$ for any $b: A_{n} \rightarrow \mathbb{R}$. We extend $p_{n}$ linearly to $\mathcal{Q}$.

Let $\mathcal{O}(n)$ be the group of (real) orthogonal $n \times n$ matrices. The group $\mathcal{O}(n)$ acts (linearly) on $\mathbb{R}\left[y_{1}, \ldots, y_{n}\right]$, and via the bijection

$$
x_{\alpha} \leftrightarrow \prod_{i \in \alpha} y_{i}
$$

between the variables $x_{\alpha}$ and monomials in $\mathbb{R}\left[y_{1}, \ldots, y_{n}\right], \mathcal{O}(n)$ also acts on $\mathbb{R}\left[x_{\alpha} \mid \alpha \in A_{n}\right]$. Then, by the First Fundamental Theorem of invariant theory for the orthogonal group $\mathcal{O}(n)$ (cf. Goodman and Wallach [2]), we have

$$
p_{n}(\mathcal{Q})=\mathbb{R}\left[x_{\alpha} \mid \alpha \in A_{n}\right]^{\mathcal{O}(n)}
$$

(The latter denotes as usual the set of polynomials in $\mathbb{R}\left[x_{\alpha} \mid \alpha \in A_{n}\right]$ invariant under $\mathcal{O}(n)$.) This can be seen using the connection (8).

\section{Derivatives}

For any polynomial $p \in \mathbb{R}\left[x_{\alpha} \mid \alpha \in A_{n}\right]$, let $d p$ be its derivative, being an element of $\mathbb{R}\left[x_{\alpha} \mid \alpha \in A_{n}\right] \otimes_{\mathbb{R}} L_{n}$, where $L_{n}$ is the space of linear functions in $\mathbb{R}\left[x_{\alpha} \mid \alpha \in A_{n}\right]$. Then $d^{k} p \in \mathbb{R}\left[x_{\alpha} \mid \alpha \in A_{n}\right] \otimes_{\mathbb{R}} L_{n}^{\otimes k}$. 
Let $\langle.,$.$\rangle be the inner product on L_{n}$ given by

$$
\left\langle x_{\alpha}, x_{\beta}\right\rangle:=c_{\alpha} \delta_{\alpha, \beta}
$$

for $\alpha, \beta \in A_{n}$, where

$$
c_{\alpha}:=\prod_{i=1}^{n} \mu_{i}(\alpha) !
$$

where $\mu_{i}(\alpha)$ denotes the multiplicity of $i$ in $\alpha$. This induces an inner product on $L_{n}^{\otimes k}$ for each $k$. With the usual product of polynomials in $\mathbb{R}\left[x_{\alpha} \mid \alpha \in A_{n}\right]$, this gives an inner product on $\mathbb{R}\left[x_{\alpha} \mid \alpha \in A_{n}\right] \otimes_{\mathbb{R}} L_{n}^{\otimes k}$ with values in $\mathbb{R}\left[x_{\alpha} \mid \alpha \in A_{n}\right]$.

The following lemma is basic to our proof, and is used several times in it.

Lemma 1. For all graphs $G, H$ and $k, n \in \mathbb{N}$ :

$$
p_{n}\left(\lambda_{k}(G, H)\right)=\left\langle d^{k} p_{n}(G), d^{k} p_{n}(H)\right\rangle .
$$

Proof. We expand $d^{k} p_{n}(G)$ :

$$
\begin{aligned}
& d^{k} p_{n}(G)=\sum_{\alpha_{1}, \ldots, \alpha_{k} \in A_{n}} \sum_{\phi: E G \rightarrow[n]} \frac{d}{d x_{\alpha_{1}}} \cdots \frac{d}{d x_{\alpha_{k}}}\left(\prod_{v \in V G} x_{\phi(\delta(v))}\right) \otimes x_{\alpha_{1}} \otimes \cdots \otimes x_{\alpha_{k}}= \\
& \sum_{\alpha_{1}, \ldots, \alpha_{k} \in A_{n}} \sum_{\phi: E G \rightarrow[n]} \sum_{\substack{u_{1}, \ldots, u_{k} \in V G \\
\forall i: \phi\left(\delta\left(u_{i}\right)\right)=\alpha_{i}}}\left(\prod_{v \in V G \backslash\left\{u_{1}, \ldots, u_{k}\right\}} x_{\phi(\delta(v))}\right) \otimes x_{\alpha_{1}} \otimes \cdots \otimes x_{\alpha_{k}}= \\
& \sum_{u_{1}, \ldots, u_{k} \in V G} \sum_{\phi: E G \rightarrow[n]}\left(\prod_{v \in V G \backslash\left\{u_{1}, \ldots, u_{k}\right\}} x_{\phi(\delta(v))}\right) \otimes x_{\phi\left(\delta\left(u_{1}\right)\right)} \otimes \cdots \otimes x_{\phi\left(\delta\left(u_{k}\right)\right)} .
\end{aligned}
$$

Here $u_{1}, \ldots, u_{k}$ are distinct. Now for any $\phi: E G \rightarrow[n]$ and $\psi: E H \rightarrow[n]$ and any $u \in V G$ and $v \in V H,\left\langle x_{\phi(\delta(u))}, x_{\psi(\delta(v))}\right\rangle$ is equal to the number of bijections $\pi: \delta(u) \rightarrow \delta(v)$ such that $\psi \circ \pi=\phi \mid \delta(u)$. This implies (12).

\section{Proof of Theorem 1}

To see necessity, let $b: A_{n} \rightarrow \mathbb{R}$ and $f=f_{b}$. Then, trivially, $f$ is multiplicative. Positive semidefiniteness of $M_{f, k}$ follows from

$$
f_{b}\left(\lambda_{k}(G, H)\right)=p_{n}\left(\lambda_{k}(G, H)\right)(b)=\left\langle d^{k} p_{n}(G)(b), d^{k} p_{n}(H)(b)\right\rangle,
$$

using Lemma 1. 
We next show sufficiency. First we have:

Claim 1. Let $\gamma$ be a quantum graph consisting of $k$-vertex graphs. If $f\left(\lambda_{k}(\gamma, \gamma)\right)=0$ then $f(\gamma)=0$

Proof. We prove the claim by induction on $k$. So assume that the claim holds for all quantum graphs made of graphs with less than $k$ vertices.

We can assume that all graphs occurring in $\gamma$ with nonzero coefficient have the same degree sequence $d_{1}, \ldots, d_{k}$, since if we would write $\gamma=\gamma_{1}+\gamma_{2}$, where all graphs in $\gamma_{1}$ have degree sequence different from those in $\gamma_{2}$, then $\lambda_{k}\left(\gamma_{1}, \gamma_{2}\right)=0$, whence $f\left(\lambda_{k}\left(\gamma_{i}, \gamma_{i}\right)\right)=0$ for $i=1,2$.

Now $f\left(\lambda_{k}(\gamma, \gamma)\right)=0$ implies, by the positive semidefiniteness of $M_{f, k}$ :

$$
f\left(\lambda_{k}(\gamma, H)\right)=0 \text { for each graph } H \text {. }
$$

Let $P$ be the graph with $2 k$ vertices $1,1^{\prime}, \ldots, k, k^{\prime}$, where for each $i=1, \ldots, k$, there are $d_{i}$ parallel edges connecting $i$ and $i^{\prime}$. If $d_{1}, \ldots, d_{k}$ are all distinct, we are done, since then $\gamma$ is a multiple of $\lambda_{k}(\gamma, P)$, implying with (15) that $f(\gamma)=0$ - but generally there can be vertices of equal degrees.

The sum in (5) for $\lambda_{k}(\gamma, P)$ can be decomposed according to the set $I$ of those components of $P$ with both vertices chosen among $v_{1}, \ldots, v_{k}$ and to the set $J$ of those components of $P$ with no vertices chosen among $v_{1}, \ldots, v_{k}$ (necessarily $|I|=|J|$ ). Let $K$ denote the set of components of $P$, and for $J \subseteq K$, let $P_{J}$ be the union of the components in $J$. Then

$$
\lambda_{k}(\gamma, P)=\sum_{\substack{I, J \subseteq K \\ I \cap J=\emptyset,|I|=|J|}} \alpha_{I, J} \gamma_{I} P_{J}
$$

where $\alpha_{I, J} \in \mathbb{N}$ with $\alpha_{\emptyset, \emptyset} \neq 0$, and where

$$
\gamma_{I}:=\lambda_{2|I|}\left(\gamma, P_{I}\right)
$$

Now for each $I \subseteq K$, we have $\lambda_{k-2|I|}\left(\gamma_{I}, \gamma_{I}\right)=\lambda_{k}\left(\gamma, \gamma_{I} P_{I}\right)$. Hence

$$
f\left(\lambda_{k-2|I|}\left(\gamma_{I}, \gamma_{I}\right)\right)=f\left(\lambda_{k}\left(\gamma, \gamma_{I} P_{I}\right)\right)=0
$$

by (15). So by induction, if $I \neq \emptyset$ then $f\left(\gamma_{I}\right)=0$. Therefore, by (16), since $f\left(\lambda_{k}(\gamma, P)\right)=0$ and $\alpha_{\emptyset, \emptyset} \neq 0, f(\gamma)=f\left(\gamma_{\emptyset} P_{\emptyset}\right)=0$.

Let $O$ be the graph just consisting of the pointless loop.

Claim 2. $f(O) \in \mathbb{N}$.

Proof. Suppose not. Then we can choose a $k \in \mathbb{N}$ with $\left(\begin{array}{c}f(O) \\ k\end{array}\right)<0$. For each $\pi \in S_{k}$, let $G_{\pi}$ be the graph with vertex set $[k]$ and edges $\{i, \pi(i)\}$ for $i=1, \ldots, k$. (So $G_{\pi}$ is 2-regular.) Define 


$$
\gamma:=\sum_{\pi \in S_{k}} \operatorname{sgn}(\pi) G_{\pi}
$$

Then for any $n$ :

$$
\begin{aligned}
& p_{n}(\gamma)=\sum_{\pi \in S_{k}} \operatorname{sgn}(\pi) p_{n}\left(G_{\pi}\right)=\sum_{\pi \in S_{k}} \operatorname{sgn}(\pi) \sum_{\phi: E G_{\pi} \rightarrow[n]} \prod_{v \in V G_{\pi}} x_{\phi(\delta(v))}= \\
& \sum_{\pi \in S_{k}} \operatorname{sgn}(\pi) \sum_{\phi:[k] \rightarrow[n]} \prod_{v \in[k]} x_{\left\{\phi(v), \phi \circ \pi^{-1}(v)\right\}}=\sum_{\phi:[k] \rightarrow[n]} \sum_{\pi \in S_{k}} \operatorname{sgn}(\pi) \prod_{v \in[k]} x_{\{\phi(v), \phi \circ \pi(v)\}} .
\end{aligned}
$$

Now if $\phi$ is not injective, then $\phi=\phi \circ \pi$ for some $\pi \in S_{k}$ with $\operatorname{sgn}(\pi)=-1$, and hence the last inner sum is 0 . So if $n<k$ then $p_{n}(\gamma)=0$. If $n=k$, then $p_{n}(\gamma)$ contains the term $x_{\{1,1\}} \cdots x_{\{k, k\}}$ with nonzero coefficient, so $p_{k}(\gamma) \neq 0$.

Since $\lambda_{k}(\gamma, \gamma)$ is a sum of graphs with no vertices, we know that $\lambda_{k}(\gamma, \gamma)=q(O)$ for some polynomial $q \in \mathbb{R}[y]$, of degree at most $k$. Then, if $n<k$,

$$
q(n)=q\left(p_{n}(O)\right)=p_{n}(q(O))=p_{n}\left(\lambda_{k}(\gamma, \gamma)\right)=0
$$

with Lemma 1, as $p_{n}(\gamma)=0$. Moreover, $q(k)=p_{k}\left(\lambda_{k}(\gamma, \gamma)\right)>0$, as $p_{k}(\gamma) \neq 0$ (again using Lemma 1). So $q(y)=c\left(\begin{array}{l}y \\ k\end{array}\right)$ for some $c>0$. Therefore,

$$
f\left(\lambda_{k}(\gamma, \gamma)\right)=f(q(O))=q(f(O))=c\left(\begin{array}{c}
f(O) \\
k
\end{array}\right)<0,
$$

contradicting the positive semidefiniteness condition.

This gives us $n$ :

$$
n:=f(O)
$$

Then Claim 1 implies:

$$
\text { there is linear function } \hat{f}: p_{n}(\mathcal{Q}) \rightarrow \mathbb{R} \text { such that } f=\hat{f} \circ p \text {. }
$$

Otherwise, there is a quantum graph $\gamma$ with $p_{n}(\gamma)=0$ and $f(\gamma) \neq 0$. We can assume that $p_{n}(\gamma)$ is homogeneous, that is, all graphs in $\gamma$ have the same number of vertices, $k$ say. Hence, since $\lambda_{k}(\gamma, \gamma)$ is a polynomial in $O$, and since $f(O)=n=p_{n}(O)$,

$$
f\left(\lambda_{k}(\gamma, \gamma)\right)=p_{n}\left(\lambda_{k}(\gamma, \gamma)\right)=0
$$

by Lemma 1 . So by Claim $1, f(\gamma)=0$. This proves $(24)$.

In fact, $\hat{f}$ is an algebra homomorphism, since for all $G, H \in \mathcal{G}$ :

$$
\hat{f}\left(p_{n}(G) p_{n}(H)\right)=\hat{f}\left(p_{n}(G H)\right)=f(G H)=f(G) f(H)=\hat{f}\left(p_{n}(G)\right) \hat{f}\left(p_{n}(H)\right) .
$$


Then for all $G, H \in \mathcal{G}$, using Lemma 1 :

$$
\hat{f}\left(\left\langle d p_{n}(G), d p_{n}(H)\right\rangle\right)=\hat{f}\left(p_{n}\left(\lambda_{1}(G, H)\right)\right)=f\left(\lambda_{1}(G, H)\right)=\left(M_{f, 1}\right)_{G, H} .
$$

Since $M_{f, 1}$ is positive semidefinite, $(27)$ implies that for each $q \in p_{n}(\mathcal{Q})$ :

$$
\hat{f}(\langle d q, d q\rangle) \geq 0
$$

Now choose $\Delta \in \mathbb{N}$. Let $\mathcal{G}_{\Delta}$ be the set of graphs of maximum degree at most $\Delta$, and let $\mathcal{Q}_{\Delta}$ be the set of all formal linear combinations of graphs in $\mathcal{G}_{\Delta}$. Define

$$
A_{n, \Delta}:=\left\{\alpha \in A_{n}|| \alpha \mid \leq \Delta\right\}
$$

By (28) and since the inner product $\langle.,$.$\rangle on L_{n}$ is $\mathcal{O}(n)$-invariant, the theorem of Procesi and Schwarz [4] (which we can apply in view of (9)) implies the existence of a $b \in \mathbb{R}^{A_{n, \Delta}}$ such that $\hat{f}(p)=p(b)$ for each $p \in p_{n}\left(\mathcal{Q}_{\Delta}\right)$. So

$$
f(G)=\hat{f}\left(p_{n}(G)\right)=p_{n}(G)(b)=f_{b}(G)
$$

for each $G \in \mathcal{G}_{\Delta}$.

This can be extended to the collection $\mathcal{G}$ of all graphs. For each $d$, let $K_{2}^{(d)}$ be the graph with two vertices, connected by $d$ parallel edges. Then any $b: A_{n} \rightarrow \mathbb{R}$ with $f=f_{b}$ satisfies

$$
b(\alpha)^{2} \leq f\left(K_{2}^{(|\alpha|)}\right)
$$

for $\alpha \in A_{n}$.

For each $\Delta \in \mathbb{N}$, define

$$
\begin{aligned}
& B_{\Delta}:=\left\{b: A_{n} \rightarrow \mathbb{R} \mid f(G)=f_{b}(G) \text { for each } G \in \mathcal{G}_{\Delta}, b(\alpha)^{2} \leq f\left(K_{2}^{(|\alpha|)}\right)\right. \text { for each } \\
& \left.\alpha \in A_{n}\right\} .
\end{aligned}
$$

By the above, $B_{\Delta} \neq \emptyset$ for each $\Delta$. As each $B_{\Delta}$ is compact by Tychonoff's theorem, and as $B_{\Delta} \supseteq B_{\Delta^{\prime}}$ if $\Delta \leq \Delta^{\prime}$, we know $\bigcap_{\Delta} B_{\Delta} \neq \emptyset$. Any $b$ in this intersection satisfies $f=f_{b}$.

Note that the positive semidefiniteness of $M_{f, k}$ for $k \neq 1$ is only used to prove Claims 2 and 1. If we know that $f=\hat{f} \circ p_{n}$ for some linear function $\hat{f}: p_{n}(\mathcal{Q}) \rightarrow \mathbb{R}$ and some $n$, then it suffices to require that $M_{f, 1}$ is positive semidefinite and $f$ is multiplicative.

\section{Derivation of Szegedy's theorem}

We now derive as a consequence the theorem of Szegedy [5]. Consider some $k \in \mathbb{N}$. A $k$-exit graph is a pair $(G, u)$ of an undirected graph $G$ and an element $u \in V G^{k}$ such that the $u_{i}$ are distinct vertices, each of degree 1 . Let $\mathcal{G}_{k}$ denote the collection of $k$-exit graphs. 
If $(G, u)$ and $(H, v)$ are $k$-exit graphs, then $(G, u) \cdot(H, v)$ is the undirected graph obtained by taking the disjoint union of $G$ and $H$, and, for each $i=1, \ldots, k$, deleting $u_{i}$ and $v_{i}$ and adding a new point connecting the ends left by $u_{i}$ and $v_{i}$. by

Let $\mathcal{G}_{k}$ be the collection of $k$-exit graphs. For $f: \mathcal{G} \rightarrow \mathbb{R}$, define the $\mathcal{G}_{k} \times \mathcal{G}_{k}$ matrix $N_{f, k}$

$$
\left(N_{f, k}\right)_{(G, u),(H, v)}:=f((G, u) \cdot(H, v))
$$

for $(G, u),(H, v) \in \mathcal{G}_{k}$. Then $([5])$ :

Corollary 1a. Let $f: \mathcal{G} \rightarrow \mathbb{R}$. Then $f=f_{b}$ for some $n \in \mathbb{N}$ and some $b: A_{n} \rightarrow \mathbb{R}$ if and only if $f$ is multiplicative and $N_{f, k}$ is positive semidefinite for each $k \in \mathbb{N}$.

Proof. Necessity follows similarly as in Theorem 1. To see sufficiency, let for any graph $G$ and any $d \in \mathbb{N}, G_{d}$ be the quantum $d$-exit graph being the sum of all $d$-exit graphs obtained as follows. Choose a vertex $v$ of degree $d$, delete $v$ topologically from $G$, and add vertices of degree 1 to the loose ends. Let $F$ be the graph obtained this way. Order these new vertices in a vector in $u \in V F^{d}$. The sum of these $(F, u)$ makes $G_{d}$. So $G_{d}$ is a sum of precisely $d ! m$ $d$-exit graphs, where $m$ is the number of vertices of degree $d$ in $G$.

We can repeat this to define the quantum $d_{1}+\cdots+d_{k}$-exit graph $G_{d_{1}, \ldots, d_{k}}$ for any $d_{1}, \ldots, d_{k} \in \mathbb{N}$, where we concatenate the exit vectors. Then

$$
\lambda_{k}(G, H)=\sum_{d_{1}, \ldots, d_{k}} c_{d_{1}, \ldots, d_{k}} G_{d_{1}, \ldots, d_{k}} \cdot H_{d_{1}, \ldots, d_{k}}
$$

for some $c_{d_{1}, \ldots, d_{k}}>0$ (namely, the inverse of the number of permutations $\pi \in S_{k}$ with $d_{\pi(i)}=d_{i}$ for each $\left.i \in[k]\right)$. Hence for any quantum graph $\gamma$ :

$$
f\left(\lambda_{k}(\gamma, \gamma)\right)=\sum_{d_{1}, \ldots, d_{k}} c_{d_{1}, \ldots, d_{k}} f\left(\gamma_{d_{1}, \ldots, d_{k}} \cdot \gamma_{d_{1}, \ldots, d_{k}}\right) \geq 0
$$

by the positive semidefiniteness of the $N_{f, l}$.

\section{Uniqueness of $b$}

We finally consider the uniqueness of $b$, and extend a theorem of Szegedy [5] (who showed sufficiency). As usual, $b^{U}$ denotes the result of the action of $U$ on $b$.

Theorem 2. Let $b: A_{n} \rightarrow \mathbb{R}$ and $c: A_{m} \rightarrow \mathbb{R}$. Then $f_{b}=f_{c}$ if and only if $n=m$ and $c=b^{U}$ for some $U \in \mathcal{O}(n)$.

Proof. Sufficiency can be seen as follows. Let $n=m$ and $c=b^{U}$ for some $U \in \mathcal{O}(n)$. Then for any graph $G$, using (9),

$$
f_{b}(G)=p_{n}(G)(b)=p_{n}(G)^{U^{-1}}(b)=p_{n}(G)\left(b^{U}\right)=f_{b^{U}}(G)=f_{c}(G)
$$


Conversely, let $f_{b}(G)=f_{c}(G)$ for each graph $G$. Then $n=f_{b}(O)=f_{c}(O)=m$. We show that for each $\Delta \in \mathbb{N}$, there exists $U \in \mathcal{O}(n)$ such that $c\left|A_{n, \Delta}=b^{U}\right| A_{n, \Delta}$, where $A_{n, \Delta}$ is as in (29). As $\mathcal{O}(n)$ is compact, this implies that there exists $U \in \mathcal{O}(n)$ with $c=b^{U}$.

Suppose that $c\left|A_{n, \Delta} \neq b^{U}\right| A_{n, \Delta}$ for each $U \in \mathcal{O}(n)$. Then the sets

$$
S:=\left\{b^{U}\left|A_{n, \Delta}\right| U \in \mathcal{O}(n)\right\} \text { and } T:=\left\{c^{U}\left|A_{n, \Delta}\right| U \in \mathcal{O}(n)\right\}
$$

are compact and disjoint subsets of $\mathbb{R}^{A_{n, \Delta}}$. So, by the Stone-Weierstrass theorem, there exists a polynomial $q \in \mathbb{R}\left[x_{\alpha} \mid \alpha \in A_{n, \Delta}\right]$ such that $q(s) \leq 0$ for each $s \in S$ and $q(t) \geq 1$ for each $t \in T$. Replacing $q$ by

$$
\int_{\mathcal{O}(n)} q^{U} d \mu(U)
$$

(where $\mu$ is the normalized Haar measure on $\mathcal{O}(n)$ ), we can assume that $q^{U}=q$ for each $U \in \mathcal{O}(n)$. Hence by $(9), q \in p_{n}(\mathcal{Q})$, say $q=p_{n}(\gamma)$ with $\gamma \in \mathcal{Q}$. Then $f_{b}(\gamma)=p_{n}(\gamma)(b)=$ $q(b) \leq 0$ and $f_{c}(\gamma)=p_{n}(\gamma)(c)=q(c) \geq 1$. This contradicts $f_{b}=f_{c}$.

Acknowledgement. I am indebted to Jan Draisma for pointing out reference [4] to me.

\section{References}

[1] M.H. Freedman, L. Lovász, A. Schrijver, Reflection positivity, rank connectivity, and homomorphisms of graphs, Journal of the American Mathematical Society 20 (2007) 37-51.

[2] R. Goodman, N.R. Wallach, Representations and Invariants of the Classical Groups, Cambridge University Press, Cambridge, 1998.

[3] P. de la Harpe, V.F.R. Jones, Graph invariants related to statistical mechanical models: examples and problems, Journal of Combinatorial Theory, Series B 57 (1993) 207-227.

[4] C. Procesi, G. Schwarz, Inequalities defining orbit spaces, Inventiones Mathematicae 81 (1985) $539-554$.

[5] B. Szegedy, Edge coloring models and reflection positivity, Journal of the American Mathematical Society 20 (2007) 969-988. 